\title{
A METHOD OF MAKING A CAST IN SYNTHETIC RESIN OF THE BRONCHIAL ARTERIES
}

\author{
BY \\ D. H. TOMPSETT \\ From the Royal College of Surgeons of England, London
}

(RECEIVED FOR PUBLICATION NOVEMBER 18, 1953)

A corrosion cast of the bronchial arteries provides a useful method of demonstrating their origin and distribution. A cast of the bronchial tree is made at the same time to show the position of the arteries. The cast produced by the method described here is limited to the principal branches of these arteries, the finer terminal twigs being lost. However, as these terminal twigs follow closely the branches of the bronchial tree, this defect is not important.

The technique described below for preparing a cast of the bronchial arteries and the bronchial tree gives only the details for the injection of the arteries, as the technique for preparing a cast of the bronchial tree has been fully described (Tompsett, 1952).

\section{MATERIAL}

The lungs and heart are removed en masse at necropsy, with the first three inches of the descending aorta. The aorta must be carefully dissected away from the thoracic wall, together with the stumps of the intercostal vessels. If it is dragged away there is a risk that the intercostal vessels may break off at their point of origin from the aorta, with the loss of any bronchial twigs that may arise from them.

\section{Fixation OF MATERIAL}

The left atrium is opened and the ventricles and right atrium are removed by cutting round the junction between the left atrium and the rest of the heart, and by cutting through the pulmonary artery and aorta as close to the heart as possible. Short pieces of flanged glass tubing are tied very securely into the pulmonary artery and both ends of the aorta and into the trachea. All vessels given off by the aorta excep $i$ the bronchial arteries are ligatured.

It is necessary to wash out the blood from the whole specimen as completely as possible before fixation. During this process it is important to avoid trapping air in any of the arteries. First about 5 litres of $2 \%$ sodium citrate are run into the pulmonary artery from a funnel held about a foot above the lungs. The citrate diffuses throughout the whole specimen and maintains the blood in as fluid a state as possible. Then cold tap water is run at moderate pressure from the tap into the pulmonary artery, until the fluid escaping from the pulmonary veins is nearly colourless. Next, one end of the aorta is closed by means of a piece of rubber tubing with a screw clamp, attached to the glass tube previously inserted, and tap water is run into the other end for about an hour. The tap is adjusted to produce a moderate pressure of water in the aorta. Care must be taken to avoid trapping air in the aorta, as if this were forced into the bronchial arteries it would obstruct the subsequent injection with resin. At this stage a check is made to see if there are any leaks in the aorta, such as from inter-costal vessels which have been overlooked. Such leaks are revealed by a jet of water escaping from them. Finally tap water is run into the trachea and allowed to escape via the vessels and pleura after diffusing through the lung tissue, to wash any mucus in the bronchi into the alveoli. The lungs are then de-aerated and fixed in spirit (Tompsett, 1952).

The lungs are left in spirit for a week, but after the first 24 hours some spirit is forced into one end of the aorta with the other end closed by means of an enema syringe. This dislodges and disintegrates any residual blood which otherwise tends to form very solid clots.

\section{INJECTION OF THE LUNGS WITH RESIN}

The day before the injection of the resin is to be made the lungs are removed from spirit and immersed in water. Tap water is run through the bronchi for several hours to remove the spirit from them. Then water is run into the aorta for half an hour at moderate pressure to wash out the bronchial arteries, and to counteract the tendency of the spirit to contract them. Finally the lungs are again de-aerated, and the apparatus arranged for the injection.

The injection of the bronchial arteries is done by using a glass tube of about $\frac{3}{4}$ in. internal diameter and about $2 \mathrm{ft}$. long as a reservoir for the resin. This tube is connected to the cut end of the descending aorta. The flow of resin is assisted by means of an enema syringe, which is attached to the upper end of the glass tube containing the resin by means of a rubber bung with a hole through it. When the enema syringe is being used this bung is held in position by hand, so that it can be quickly removed 
if it is necessary to top up the resin in the glass tube.

When the enema syringe is being used the exerition of excessive pressure is avoided by an assistant placing a finger on the aorta to feel its turgidity. It is not necessary to use a manometer, but the ends of all glass tubing must be flanged, and all connexions between glass and rubber tubing must be very securely tied on, as otherwise the pressure exerted by the enema syringe will disconnect them. Fig. 1 shows a diagram of the apparatus used for making the double injection of bronchial tree and bronchial arteries.

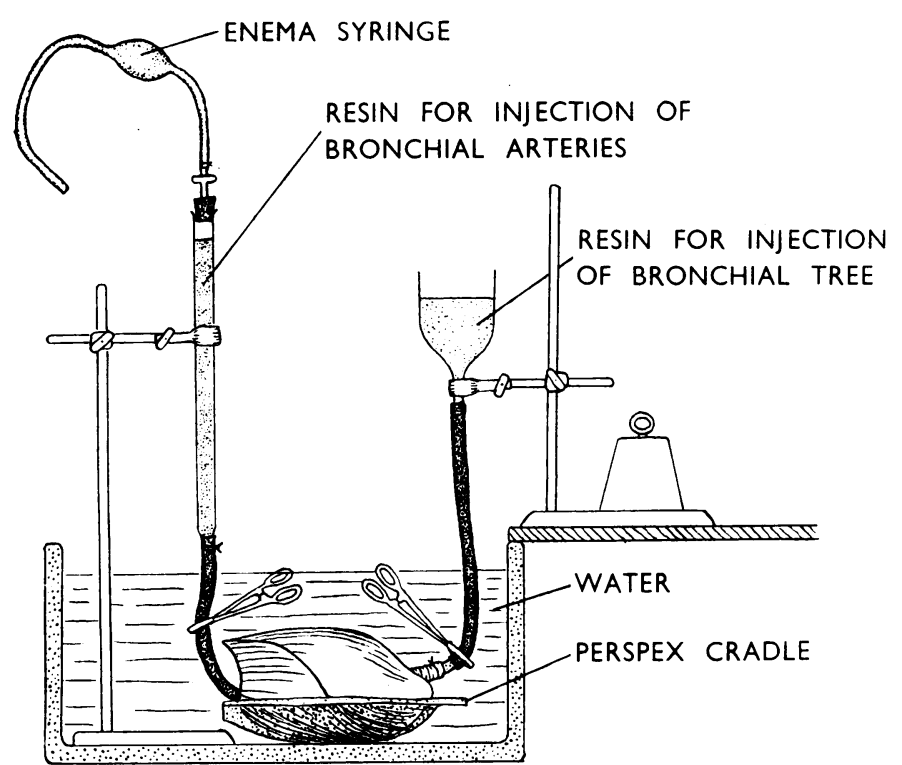

FIG. 1.-Apparatus for injection of bronchial tree and bronchial arteries.
At a room temperature of $20^{\circ} \mathrm{C}$. this has an approximate working life of 14 minutes. This is a rather more rapidly setting mixture than that recommended previously.

Of the following mixture $400 \mathrm{ml}$. is prepared for the injection of the bronchial arteries

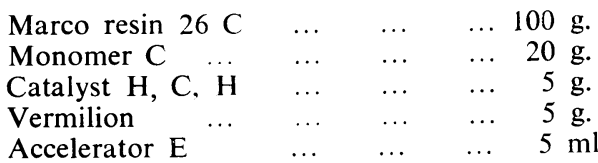

At $20^{\circ} \mathrm{C}$. this has an approximate work- $\omega$ ing life of 11 minutes. As soon as the N resin in the aorta has solidified, and 6 before it has become really hard, the 윽 rubber tubing connecting the aorta to $\rightarrow$ the injection tube (and the resin within os it) is cut through with large scissors. This $\frac{\mathbb{D}}{0}$ must be done without subjecting the aorta $\frac{\mathbb{D}}{D}$ to any dragging or twisting, as otherwise 3 the cast of the bronchial arteries may be $\mathbb{D}$ broken. The rubber tubing at the other end of the aorta is also cut through to lessen the weight of the resin cast. For $\perp$ full details concerning the injection of the bronchial tree the reader is referred to the previous paper.

\section{MACERATION AND FINISHING}

During maceration in concentrated hydrochloric acid the lungs are placed on $\frac{0}{3}$ a perspex cradle, which can be made by pressing a sheet of perspex which has been heated to $150^{\circ} \mathrm{C}$. in an oven between positive and negative plaster moulds. These moulds are heated to about $80^{\circ} \mathrm{C}$. 응

Owing to the fact that the setting of the resin is inhibited by water, a rapidly setting resin mixture must be used, and run in not more than five minutes before the resin is expected to set. If the tendency for water inhibition is not counteracted in this way, when such slender vessels as the bronchial arteries are filled with resin, the resin fails to harden properly, owing to the effect of the water in the walls of the arteries. Careful tests are made to establish the setting time of the clear resin used to fill the bronchial tree and of the coloured resin used to fill the bronchial arteries so that the injection can be correctly timed, and the accelerator is added to the two mixtures of resin at such times that they will set at the same time, the injection of the bronchi and arteries being begun simultaneously.

For the injection of the bronchial tree $600 \mathrm{ml}$. of the following resin mixture is prepared:

$\begin{array}{llllrl}\text { Marco resin } 26 \mathrm{C} & \ldots & \ldots & \ldots & 100 \mathrm{~g} . \\ \text { Monomer C } \ldots & \ldots & \ldots & \ldots & 12 \mathrm{~g} . \\ \text { Catalyst H, C, H } & \ldots & \ldots & \ldots & 4 \mathrm{~g} . \\ \text { Accelerator E } & \ldots & \ldots & \ldots & 4 \mathrm{ml} \text {. }\end{array}$
so that they do not cool the perspex too rapidly. $\times$ Fig. 2 shows a photograph of the perspex cradle, $\sigma$ which is shaped like the posterior thoracic wall.

The lungs are left in acid for three days. Ato first they float, but they sink as maceration proceeds.

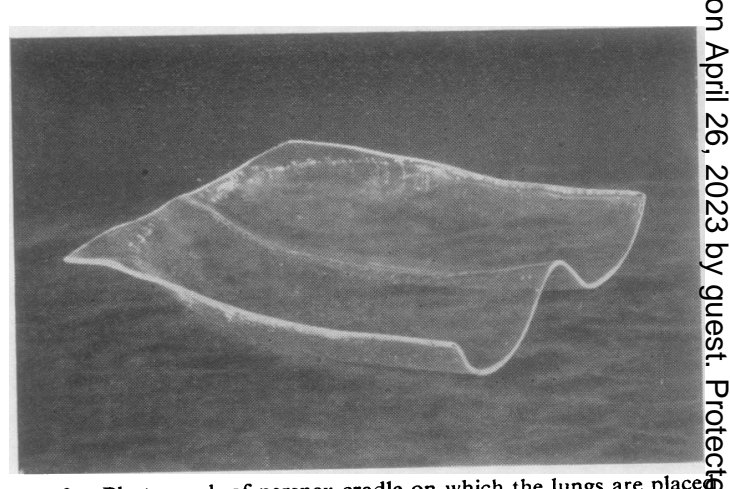

FIG. 2.-Photograph of perspex cradle on which the lungs are place during maceration in acid. 
After maceration the cast is lifted out of the acid on the cradle and immersed in cold water. The macerated tissue is washed away by means of a jet of water. This work must be done very gently and carefully, as the cast of the aorta is held in position only by the cast of the bronchial arteries, which in turn are only lying against, and not actually adhering to, the bronchial tree.

When the cast has been washed clean, the stump of the aorta is firmly fixed to the main left bronchus. This is done by shaping a small block of resin with files until it fits the gap between the bronchus and the aorta, and cementing it in position with resin $28 \mathrm{C}$.

After the bronchial tree has been pruned in the usual way, surplus resin at the two ends of the cast of the aorta is sawn off and the cast is sprayed with resin. On setting this fixes the finer branches of the bronchial arteries to the cast of the tree. Before the resin spray has set any of the finer branches of the bronchial arteries which have become displaced are arranged in their correct position. Fig. 3 shows a photograph of a cast of the bronchial tree and bronchial arteries. In this specimen there are two bronchial arteries, a relatively small one, arising together with the second right intercostal, and supplying only lymph glands and part of the upper right lobe; and a relatively larger one, arising with the third right intercostal artery, and supplying the rest of the lymph glands and lungs. In the photograph only the cast of the larger artery can be seen, as the smaller one is obscured by the cast of the right main bronchus. There is an anastomosis between these two bronchial arteries just above the right main bronchus.

\section{Discussion}

The technique for making casts of the bronchial tree is completely reliable, when skilfully applied, giving a cast which by ordinary standards can be described as perfect; but this technique should be completely mastered before it is attempted to combine it with a cast of the bronchial arteries.

The use of Marco resin for making casts of vessels as fine as the bronchial arteries is complicated by the fact that contact with water inhibits the setting of the surface layer. When a cast of a relatively large structure is made this inhibition does not appreciably affect the final cast. The inhibition also extends less deeply into the surface of the resin, as a considerable amount of heat is generated during the latter part of the setting process, and this heat reduces the depth to which inhibition extends. When fine vessels are filled, if the inhibition extends to a depth equal to the radius of the vessel injected, no solid rigid cast will be produced, no matter how long the resin is left to set. This inhibition is reduced by three means: the use of a rapidly setting mixture, containing a large percentage of catalyst and accelerator; by the use of as viscous a mixture as is
FiG. 3.-Photograph of a cast of the bronchial tree and bronchial arteries, the latter being coloured red $\left(x, \frac{1}{2}\right)$ 
practicable ; and by running the resin into the vessels as short a time as is possible before the setting process begins. But, in spite of these precautions, some inhibition still occurs.

Another drawback to the use of resin for the filling of fine vessels is that it is rather fragile in fine filaments. But it has two great advantages : first, that the injection of vessels with it is extremely simple, and, secondly, that once the resin has set it forms a rigid cast which will keep its shape indefinitely.

Although the cast of the bronchial arteries when made is fragile, it is very effectively protected from the risk of breakage when the cast has been cemented to the cast of the bronchial tree.
If the cast of these arteries is broken before it has been cemented to the cast of the tree, it can be repaired. The broken fragment is attached by means of a little perspex cement into its original position, and the joint is reinforced with cement made from resin $28 \mathrm{C}$ (Tompsett, 1952).

\section{SUMMARY}

A technique is described for making a cast of the bronchial arteries to show their relationship with the aorta and the bronchi.

\section{REFERENCE}

Tompsett, D. H. (1952). Thorax, 7, 78. 